Research Article

\title{
Global Asymptotic Stability of Switched Neural Networks with Delays
}

\author{
Zhenyu Lu, ${ }^{1}$ Kai $L i,{ }^{1}$ and Yan $\mathrm{Li}^{2}$ \\ ${ }^{1}$ College of Electrical and Information Engineering, Nanjing University of Information Science and Technology, \\ Nanjing 210044, China \\ ${ }^{2}$ College of Science, Huazhong Agricultural University, Wuhan 430070, China
}

Correspondence should be addressed to Zhenyu Lu; luzhenyu76@163.com

Received 1 September 2015; Accepted 30 November 2015

Academic Editor: Yuan Fan

Copyright (C) 2015 Zhenyu Lu et al. This is an open access article distributed under the Creative Commons Attribution License, which permits unrestricted use, distribution, and reproduction in any medium, provided the original work is properly cited.

\begin{abstract}
This paper investigates the global asymptotic stability of a class of switched neural networks with delays. Several new criteria ensuring global asymptotic stability in terms of linear matrix inequalities (LMIs) are obtained via Lyapunov-Krasovskii functional. And here, we adopt the quadratic convex approach, which is different from the linear and reciprocal convex combinations that are extensively used in recent literature. In addition, the proposed results here are very easy to be verified and complemented. Finally, a numerical example is provided to illustrate the effectiveness of the results.
\end{abstract}

\section{Introduction}

In the past thirty years, neural networks have found extensive applications in associative memory, pattern recognition, and image processing [1-3]. It is true that most applications of neural networks are heavily dependent on the dynamic behaviors of neural networks, especially on global asymptotic stability of neural networks. On the other hand, time delays are inevitably encountered in the hardware implementation due to the finite switching speed of amplifier, which may destroy the system performance and become a source of oscillation or instability in neural networks. Therefore, stability of neural networks with delays has attracted increasing attention and lots of stability criteria have been reported in the literature $[4,5]$.

As a special class of hybrid systems, switched systems are organized by a switching rule that orchestrates the switching. In reality, neural networks sometimes have finite modes that switch from one to another at different times according to a switching law. In $[6,7]$, the authors studied the stability problem of different kinds of switched neural networks with time delays. Different from the model in these works, in this paper, we consider a class of neural networks with state-dependent switchings. Our switched neural networks model is general and it generalizes the conventional neural networks.

Recently, convex analysis has been significantly employed in the stability analysis of time-delay systems [8-17]. According to the feature of different convex functions, different convex combination approaches are adopted in the literature, such as the linear convex combination [8-10], reciprocal convex combination [11-13], and quadratic convex combination [15-17]. In $[8,9,12,14,16,17]$, convex combination technology was successfully used to derive some stability criteria for neural networks with time delays. It should be pointed out that the lower bound of the time delay in $[8,9,16]$ is zero, which means the information on the lower bound of the time delay cannot be sufficiently used. Namely, the conditions obtained in $[9,14,16]$ fail to take effect on the stability of neural networks when the lower bound of the time delay is strictly greater than zero.

In this paper, some delay-dependent stability criteria in terms of LMIs are derived. The advantages are as follows. Firstly, differential inclusions and set-valued maps are 
employed to deal with the switched neural networks with discontinuous right-hand sides. Secondly, our results employ the quadratic convex approach, which is different from the linear and reciprocal convex combinations that are extensively used in recent literature on stability. Thirdly, the lower bound $\tau_{1}$ of the time-varying delays is not zero and its information is adequately used to construct the Lyapunov-Krasovskii functional. Fourthly, we resort to neither Jensen's inequality with delay-dividing approach nor the free-weighting matrix method compared with previous results.

The organization of this paper is as follows. Some preliminaries are introduced in Section 2. In Section 3, based on the quadratic convex approach, delay-dependent stability criteria in terms of LMIs are established for switched neural networks with time-varying delays. Then, an example is given to demonstrate the effectiveness of the obtained results in Section 4. Finally, conclusions are given in Section 5.

Notations. Throughout this paper, $R^{n}$ denotes the $n$-dimensional Euclidean space. $A^{T}$ and $A^{-1}$ denote the transpose and the inverse of the matrix $A$, respectively. $A>0(A \geq 0)$ means that the matrix $A$ is symmetric and positive definite (semipositive definite). $*$ represents the elements below the main diagonal of a symmetric matrix. The identity and zero matrices of appropriate dimensions are denoted by $I$ and 0 , respectively. $\operatorname{SYM}(A)$ is defined as $\operatorname{SYM}(A)=A+A^{T}$. $\operatorname{diag}\{\cdots\}$ denotes a block-diagonal matrix.

\section{System Description and Preliminaries}

In this paper, we consider a class of switched neural networks with delays as follows:

$$
\begin{array}{r}
\dot{x}_{i}(t)=-d_{i}\left(x_{i}(t)\right) x_{i}(t)+\sum_{j=1}^{n} a_{i j}\left(x_{i}(t)\right) f_{j}\left(x_{j}(t)\right) \\
+\sum_{j=1}^{n} b_{i j}\left(x_{i}(t)\right) f_{j}\left(x_{j}\left(t-\tau_{j}(t)\right)\right), \\
t \geq 0, i=1,2, \ldots, n,
\end{array}
$$

where

$$
\begin{aligned}
& d_{i}\left(x_{i}(t)\right)= \begin{cases}d_{i}^{*}, & x_{i}(t) \leq 0, \\
d_{i}^{* *}, & x_{i}(t)>0,\end{cases} \\
& a_{i j}\left(x_{i}(t)\right)= \begin{cases}a_{i j}^{*}, & x_{i}(t) \leq 0, \\
a_{i j}^{* *}, & x_{i}(t)>0,\end{cases} \\
& b_{i j}\left(x_{i}(t)\right)= \begin{cases}b_{i j}^{*}, & x_{i}(t) \leq 0, \\
b_{i j}^{* *}, & x_{i}(t)>0,\end{cases}
\end{aligned}
$$

$x_{i}(t)$ is the state variable of the $i$ th neuron, and $a_{i j}\left(x_{i}(t)\right)$ and $b_{i j}\left(x_{i}(t)\right)$ denote the feedback connection weight and the delayed feedback connection weight, respectively. $f_{j}: R \rightarrow$ $R$ is bounded continuous function; $\tau_{j}(t)$ corresponds to the transmission delay and satisfies $i, j=1,2, \ldots, n . d_{i}^{*}>0$, $d_{i}^{* *}>0, a_{i j}^{*}, a_{i j}^{* *}, b_{i j}^{*}$, and $b_{i j}^{* *}, i, j=1,2, \ldots, n$, are all constant numbers. The initial condition of system (1) is $x(s)=\phi(s)=$ $\left(\phi_{1}(s), \phi_{2}(s), \ldots, \phi_{n}(s)\right)^{T} \in \mathscr{C}\left(\left[-\tau_{2}, 0\right], R^{n}\right)$.

The following assumptions are given for system (1):

(H1) For $j \in 1,2, \ldots, n, f_{j}$ is bounded and there exist constants $h_{j}^{-}, h_{j}^{+}$such that

$$
h_{j}^{-} \leq \frac{f_{j}\left(s_{1}\right)-f_{j}\left(s_{2}\right)}{s_{1}-s_{2}} \leq h_{j}^{+}, \quad f_{j}(0)=0,
$$

for all $s_{1}, s_{2} \in R, s_{1} \neq s_{2}$.

(H2) The transmission delay $\tau_{j}(t)$ is a differential function and there exist constants $0 \leq \tau_{1}<\tau_{2}, \mu$ such that

$$
\begin{aligned}
0 & \leq \tau_{1} \leq \tau_{j}(t) \leq \tau_{2}, \\
\dot{\tau}_{j}(t) & \leq \mu,
\end{aligned}
$$

for all $t \geq 0, j=1,2, \ldots, n$.

Obviously, system (1) is a discontinuous system; then its solution is different from the classic solution and cannot be defined in the conventional sense. In order to obtain the solution of system (1), some definitions and lemmas are given.

Definition 1. For a system with discontinuous right-hand sides,

$$
\frac{\mathrm{d} x}{\mathrm{~d} t}=F(t, x), \quad x(0)=x_{0}, x \in R^{n}, t \geq 0 .
$$

A set-valued map is defined as

$$
\Phi(t, x)=\bigcap_{\delta>0} \bigcap_{\mu(N)=0} \overline{c o}[F(t, B(x, \delta) \backslash N)]
$$

where $\overline{\mathrm{co}}[E]$ is the closure of the convex hull of set $E, E \subset R^{n}$, $B(x, \delta)=\left\{y:\|y-x\|<\delta, x, y \in R^{n}, \delta \in R^{+}\right\}$, and $N \subset R^{n}$, $\mu(N)$ is Lebesgue measure of set $N$.

A solution in Filippov's sense of system (5) with initial condition $x(0)=x_{0} \in R^{n}$ is an absolutely continuous function $x(t), t \in[0, T], T>0$, which satisfy $x(0)=x_{0}$ and differential inclusion:

$$
\frac{\mathrm{d} x}{\mathrm{~d} t} \in \Phi(t, x), \quad \text { for a.a. } t \in[0, T]
$$

If $F(t, x)$ is bounded, then the set-valued function $\Phi(t, x)$ is upper semicontinuous with nonempty, convex, and compact values [18]. Then, the solution $x(t)$ of system (5) with 
initial condition exists and it can be extended to the interval $[0,+\infty)$ in the sense of Filippov.

By applying the theories of set-valued maps and differential inclusions [18-20], system (1) can be rewritten as the following differential inclusion:

$$
\begin{aligned}
\dot{x}_{i}(t) \in & -\left[\underline{d}_{i}, \bar{d}_{i}\right] x_{i}(t)+\sum_{j=1}^{n}\left[\underline{a}_{i j}, \bar{a}_{i j}\right] f_{j}\left(x_{j}(t)\right) \\
& +\sum_{j=1}^{n}\left[\underline{b}_{i j}, \bar{b}_{i j}\right] f_{j}\left(x_{j}\left(t-\tau_{j}(t)\right)\right),
\end{aligned}
$$

for a.a. $t \geq 0, i=1,2, \ldots, n$,

where $\left[\underline{\xi}_{i}, \bar{\xi}_{i}\right]$ is the convex hull of $\left[\underline{\xi}_{i}, \bar{\xi}_{i}\right], \underline{\xi}_{i}, \bar{\xi}_{i} \in R . \underline{d}_{i}=$ $\min \left\{d_{i}^{*}, d_{i}^{* *}\right\}, \bar{d}_{i}=\max \left\{d_{i}^{*}, d_{i}^{* *}\right\}, \underline{a}_{i j}=\min \left\{a_{i j}^{*}, a_{i j}^{* *}\right\}, \bar{a}_{i j}=$ $\max \left\{a_{i j}^{*}, a_{i j}^{* *}\right\}, \underline{b}_{i j}=\min \left\{b_{i j}^{*}, b_{i j}^{* *}\right\}$, and $\bar{b}_{i j}=\max \left\{b_{i j}^{*}, b_{i j}^{* *}\right\}$. The other parameters are the same as in system (1).

Definition 2. A constant vector $x^{*}=\left(x_{1}^{*}, x_{2}^{*}, \ldots, x_{n}^{*}\right)^{T}$ is called an equilibrium point of system (1), if, for $i=1,2, \ldots, n$,

$$
\begin{aligned}
0 \in & -\left[\underline{d}_{i}, \bar{d}_{i}\right] x_{i}^{*}+\sum_{j=1}^{n}\left[\underline{a}_{i j}, \bar{a}_{i j}\right] f_{j}\left(x_{j}^{*}\right) \\
& +\sum_{j=1}^{n}\left[\underline{b}_{i j}, \bar{b}_{i j}\right] f_{j}\left(x_{j}^{*}\right) .
\end{aligned}
$$

It is easy to find that the origin $(0,0, \ldots, 0)^{T}$ is an equilibrium point of system (1).

Definition 3 (see [18]). A function $x(t)=\left(x_{1}(t), x_{2}(t)\right.$, $\left.\ldots, x_{n}(t)\right)^{T}$ is a solution of (1), with the initial condition $x(s)=$ $\phi(s)=\left(\phi_{1}(s), \phi_{2}(s), \ldots, \phi_{n}(s)\right)^{T} \in \mathscr{C}\left(\left[-\tau_{2}, 0\right], R^{n}\right)$, if $x(t)$ is an absolutely continuous function and satisfies differential inclusion (8).

Lemma 4 (see [18]). Suppose that assumption (H1) is satisfied; then solution $x(t)$ with initial condition $\phi(s)=$ $\left(\phi_{1}(s), \phi_{2}(s), \ldots, \phi_{n}(s)\right)^{T} \in \mathscr{C}\left(\left[-\tau_{2}, 0\right], R^{n}\right)$ of $(1)$ exists and it can be extended to the interval $[0,+\infty)$.

Before giving our main results, we present the following important lemmas that will be used in the proof to derive the stability conditions of the switched neural networks.

Lemma 5 (see [21]). Given constant matrices $\Sigma_{1}, \Sigma_{2}$, and $\Sigma_{3}$, where $\Sigma_{1}^{T}=\Sigma_{1}, \Sigma_{2}^{T}=\Sigma_{2}$,

$$
\left(\begin{array}{cc}
\Sigma_{1} & \Sigma_{3} \\
\Sigma_{3}^{T} & -\Sigma_{2}
\end{array}\right)<0
$$

is equivalent to the following conditions:

$$
\begin{aligned}
\Sigma_{2} & >0, \\
\Sigma_{1}+\Sigma_{3} \Sigma_{2}^{-1} \Sigma_{3}^{T} & <0 .
\end{aligned}
$$

Lemma 6 (see [17]). For real symmetric matrices $W_{1}, W_{2}$, $W_{3} \in R^{m \times m}$ and a scalar continuous function $\tau$ satisfy $\tau_{1} \leq$ $\tau \leq \tau_{2}$, where $\tau_{1}$ and $\tau_{2}$ are constants satisfying $0 \leq \tau_{1} \leq \tau_{2}$. If $W_{1} \geq 0$, then

$$
\begin{aligned}
& \tau^{2} W_{1}+\tau W_{2}+W_{3}<0, \quad \forall \tau \in\left[\tau_{1}, \tau_{2}\right] \\
& \Longleftrightarrow \tau_{i}^{2} W_{1}+\tau_{i} W_{2}+W_{3}<0, \quad(i=1,2) .
\end{aligned}
$$

Lemma 7 (see [16]). Let $W>0$, and let $y(s)$ be an appropriate dimensional vector. Then, one has the following facts for any scalar function $\eta(s) \geq 0, \forall s \in\left[t_{1}, t_{2}\right]$ :

(i)

$$
\begin{aligned}
-\int_{t_{1}}^{t_{2}} y^{T}(s) W y(s) \mathrm{d} s \leq & \left(t_{2}-t_{1}\right) \xi_{t}^{T} F_{1}^{T} W^{-1} F_{1} \xi_{t} \\
& +2 \xi_{t}^{T} F_{1}^{T} \int_{t_{1}}^{t_{2}} y(s) \mathrm{d} s,
\end{aligned}
$$

(ii)

$$
\begin{aligned}
& -\int_{t_{1}}^{t_{2}} \eta(s) y^{T}(s) W y(s) \mathrm{d} s \\
& \leq \int_{t_{1}}^{t_{2}} \eta(s) \mathrm{d} s \xi_{t}^{T} F_{2}^{T} W^{-1} F_{2} \xi_{t} \\
& \quad+2 \xi_{t}^{T} F_{2}^{T} \int_{t_{1}}^{t_{2}} \eta(s) y(s) \mathrm{d} s
\end{aligned}
$$

(iii)

$$
\begin{aligned}
& -\int_{t_{1}}^{t_{2}} \eta^{2}(s) y^{T}(s) W y(s) \mathrm{d} s \\
& \leq\left(t_{2}-t_{1}\right) \xi_{t}^{T} F_{3}^{T} W^{-1} F_{3} \xi_{t} \\
& \quad+2 \xi_{t}^{T} F_{3}^{T} \int_{t_{1}}^{t_{2}} \eta(s) y(s) \mathrm{d} s
\end{aligned}
$$

where matrices $F_{i}(i=1,2,3)$ and vector $\xi_{t}$ independent of the integral variable are appropriate dimensional arbitrary ones.

\section{Main Results}

For presentation convenience, in the following we denote $\tau_{21}=\tau_{2}-\tau_{1}, H^{-}=\operatorname{diag}\left\{h_{1}^{-}, h_{2}^{-}, \ldots, h_{n}^{-}\right\}, H^{+}=\operatorname{diag}\left\{h_{1}^{+}, h_{2}^{+}\right.$, $\left.\ldots, h_{n}^{+}\right\}, E_{i}=\left[0_{n \times(i-1) n}, I_{n}, 0_{n \times(10-i) n}\right]^{T}, i=1,2, \ldots, 10$. $A_{c}=[-D, 0,0,0,0,0,0, A, B, 0]^{T}, D=\operatorname{diag}\left(D_{i}\right)_{n \times n}, D_{i}=$ $\min \left\{\underline{d}_{i}, \bar{d}_{i}\right\}, A=\left(A_{i j}\right)_{n \times n}, A_{i j}=\max \left\{\left|\underline{a}_{i j}\right|,\left|\bar{a}_{i j}\right|\right\}, B=\left(B_{i j}\right)_{n \times n}$, $B_{i j}=\max \left\{\left|\underline{b}_{i j}\right|,\left|\bar{b}_{i j}\right|\right\}$.

Theorem 8. Suppose assumptions (H1) and (H2) hold; the origin of system (1) is globally asymptotically stable if there exist matrices $P>0, S>0, Q_{i}>0(i=1,2,3,4,5)$, $R_{j}>0(j=1,2,3,4)$, and $\Gamma=\operatorname{diag}\left(\gamma_{1}, \gamma_{2}, \ldots, \gamma_{n}\right)>0, K_{1}=$ $\operatorname{diag}\left(k_{11}, k_{12}, \ldots, k_{1 n}\right)>0, K_{2}=\operatorname{diag}\left(k_{21}, k_{22}, \ldots, k_{2 n}\right)>0$, 
and $F_{i}(i=1,2, \ldots, 12)$, such that the following two LMIs hold:

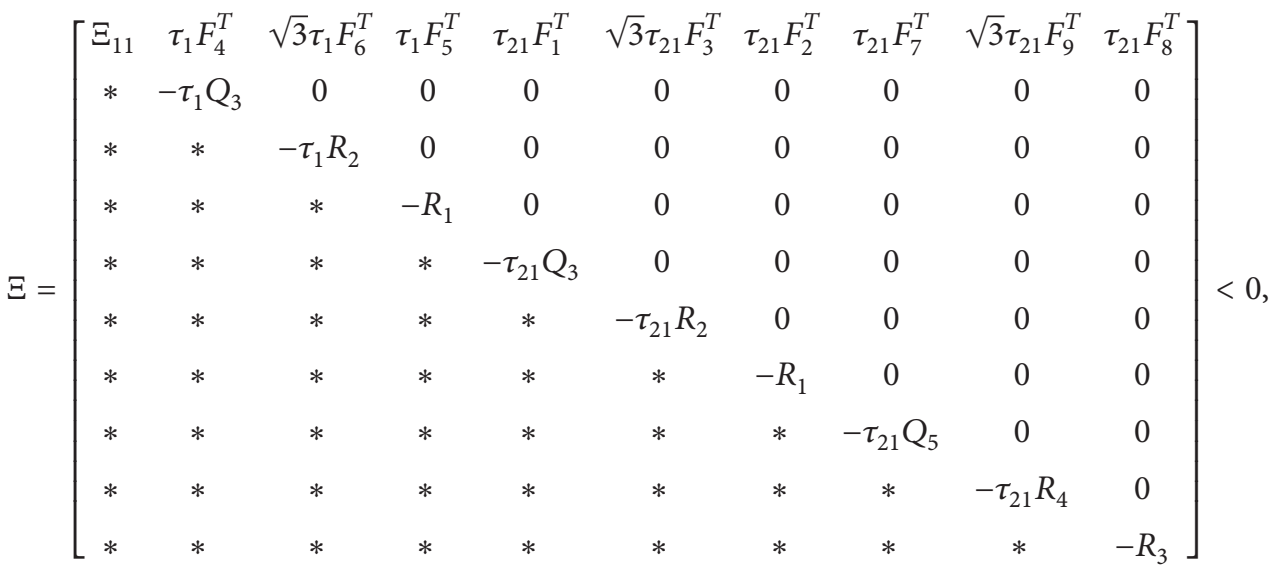

$$
\begin{aligned}
& \Pi=\left[\begin{array}{ccccccc}
\Pi_{11} & \tau_{2} F_{4}^{T} & \sqrt{3} \tau_{2} F_{6}^{T} & \tau_{2} F_{5}^{T} & \tau_{21} F_{10}^{T} & \sqrt{3} \tau_{21} F_{12}^{T} & \tau_{21} F_{11}^{T} \\
* & -\tau_{2} Q_{3} & 0 & 0 & 0 & 0 & 0 \\
* & * & -\tau_{2} R_{2} & 0 & 0 & 0 & 0 \\
* & * & * & -R_{1} & 0 & 0 & 0 \\
* & * & * & * & -\tau_{21} Q_{5} & 0 & 0 \\
* & * & * & * & * & -\tau_{21} R_{4} & 0 \\
* & * & * & * & * & * & -R_{3}
\end{array}\right]<0
\end{aligned}
$$

where $\Xi_{11}=\Delta_{0}+\operatorname{SYM}\left(\left(\tau_{21} E_{2}-E_{7}\right)\left(2 F_{2}+3 F_{3}+2 F_{8}+\right.\right.$ $\left.\left.3 F_{9}\right)\right)+\operatorname{SYM}\left(\left(\tau_{1} E_{1}-\left(E_{1}+E_{5}\right)\right)\left(2 F_{5}+3 F_{6}\right)\right), \Pi_{11}=\Delta_{0}+$ $\operatorname{SYM}\left(\left[A_{c}, 0\right] Q_{1}\left[\tau_{21} E_{1}, E_{6}\right]^{T}\right)-\operatorname{SYM}\left(E_{7}\left(2 F_{2}+3 F_{3}+2 F_{8}+\right.\right.$ $\left.\left.3 F_{9}\right)\right)+\operatorname{SYM}\left(\left(\tau_{2} E_{1}-\left(E_{5}+E_{6}\right)\right)\left(2 F_{5}+3 F_{6}\right)\right)$ with

$$
\begin{aligned}
\Delta_{0} & =\operatorname{SYM}\left(\left[E_{1}, E_{5}, E_{6}+E_{7}\right]\right. \\
& \left.\cdot P\left[A_{c}, E_{1}-E_{3}, E_{3}-E_{4}\right]^{T}+E_{8} \Gamma A_{c}^{T}\right)+E_{8} S E_{8}^{T} \\
& -(1-\mu) E_{9} S E_{9}^{T}+\left[E_{1}, E_{3}\right]\left(Q_{1}+Q_{4}\right)\left[E_{1}, E_{3}\right]^{T} \\
& -(1-\mu)\left[E_{1}, E_{2}\right] Q_{1}\left[E_{1}, E_{2}\right]^{T}+\operatorname{SYM}\left(\left[A_{c}, 0\right]\right. \\
& \cdot Q_{2}\left[\tau_{2} E_{1}, E_{5}+E_{6}+E_{7}\right]^{T}+\left[A_{c}, 0\right] \\
& \left.\cdot Q_{4}\left[\tau_{21} E_{1}, E_{6}+E_{7}\right]^{T}\right)+\left[E_{1}, E_{1}\right] Q_{2}\left[E_{1}, E_{1}\right]^{T} \\
& -\left[E_{1}, E_{4}\right]\left(Q_{2}+Q_{4}\right)\left[E_{1}, E_{4}\right]^{T}+\tau_{2}\left[E_{1}, A_{c}\right] \\
& \cdot Q_{3}\left[E_{1}, A_{c}\right]^{T}+\tau_{21}\left[E_{3}, E_{10}\right] Q_{5}\left[E_{3}, E_{10}\right]^{T} \\
+ & A_{c}\left(\tau_{2}^{2} R_{1}+\tau_{2}^{3} R_{2}\right) A_{c}^{T}+E_{10}\left(\tau_{21}^{2} R_{3}+\tau_{21}^{3} R_{4}\right) E_{10}^{T} \\
+ & S Y M\left(\left[E_{5}+E_{6}, E_{1}-E_{2}\right] F_{4}+\left[E_{7}, E_{2}-E_{4}\right] F_{7}\right. \\
+ & {\left.\left[E_{6}, E_{3}-E_{2}\right] F_{10}\right)+\operatorname{SYM}\left(E_{8} K_{1} H^{+} E_{1}^{T}\right.}
\end{aligned}
$$

$$
\begin{aligned}
& \left.+E_{1} H^{-} K_{1} E_{8}^{T}+E_{9} K_{2} H^{+} E_{2}^{T}+E_{2} H^{-} K_{2} E_{9}^{T}\right) \\
& -\operatorname{SYM}\left(E_{8} K_{1} E_{8}^{T}+E_{1} H^{-} K_{1} H^{+} E_{1}^{T}+E_{9} K_{2} E_{9}^{T}\right. \\
& \left.+E_{2} H^{-} K_{2} H^{+} E_{2}^{T}\right) .
\end{aligned}
$$

Proof. Define a vector $\xi_{t} \in R^{10 n}$ as

$$
\begin{gathered}
\xi_{t}^{T}=\left[x^{T}(t), x^{T}(t-\tau(t)), x^{T}\left(t-\tau_{1}\right), x^{T}\left(t-\tau_{2}\right),\right. \\
\int_{t-\tau_{1}}^{t} x^{T}(s) \mathrm{d} s, \int_{t-\tau(t)}^{t-\tau_{1}} x^{T}(s) \mathrm{d} s, \int_{t-\tau_{2}}^{t-\tau(t)} x^{T}(s) \mathrm{d} s, \\
\left.f^{T}(x(t)), f^{T}(x(t-\tau(t))), \dot{x}^{T}\left(t-\tau_{1}\right)\right] .
\end{gathered}
$$

Consider the following Lyapunov-Krasovskii functional candidate as follows:

$$
V(t)=V_{1}(t)+V_{2}(t)+V_{3}(t)+V_{4}(t),
$$

where

$$
\begin{aligned}
& V_{1}(t)=\zeta^{T}(t) P \zeta(t), \\
& V_{2}(t)=2 \sum_{i=1}^{n} \gamma_{i} \int_{0}^{x_{i}(t)} f_{i}(s) \mathrm{d} s,
\end{aligned}
$$




$$
\begin{aligned}
& V_{3}(t)=\int_{t-\tau(t)}^{t} f^{T}(x(s)) S f(x(s)) \mathrm{d} s
\end{aligned}
$$

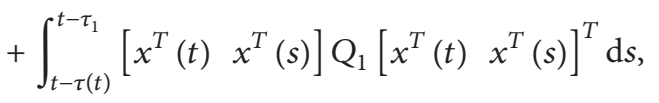

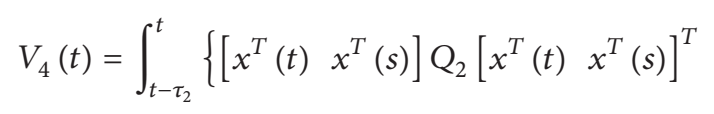

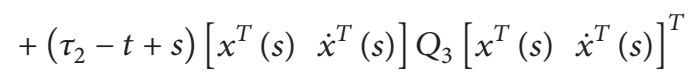

$$
\begin{aligned}
& +\left(\tau_{2}-t+s\right)^{2} \dot{x}^{T}(s) R_{1} \dot{x}(s)+\left(\tau_{2}-t+s\right)^{3} \dot{x}^{T}(s) \\
& \left.\cdot R_{2} \dot{x}(s)\right\} \mathrm{d} s
\end{aligned}
$$

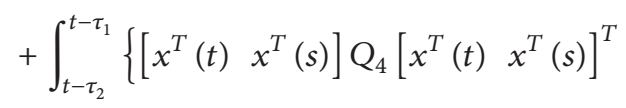

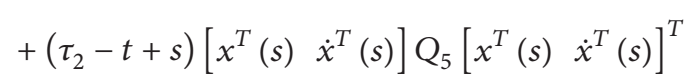

$$
\begin{aligned}
& +\left(\tau_{2}-t+s\right)^{2} \dot{x}^{T}(s) R_{3} \dot{x}(s)+\left(\tau_{2}-t+s\right)^{3} \dot{x}^{T}(s) \\
& \left.\cdot R_{4} \dot{x}(s)\right\} \mathrm{d} s,
\end{aligned}
$$

where $\zeta^{T}(t)=\left[x^{T}(t) \int_{t-\tau_{1}}^{t} x^{T}(s) \mathrm{d} s \int_{t-\tau_{2}}^{t-\tau_{1}} x^{T}(s) \mathrm{d} s\right]$.

Calculating the time derivatives of $V_{i}(t)(i=1,2,3,4)$ along the trajectories of system (8), we obtain

$$
\begin{aligned}
& \dot{V}_{1}(t)=2 \zeta^{T}(t) P \dot{\zeta}(t) \leq 2 x^{T}(t) \\
& \cdot P\left[\begin{array}{c}
-D x(t)+A f(x(t))+B f(x(t-\tau(t))) \\
x(t)-x\left(t-\tau_{1}\right) \\
x\left(t-\tau_{1}\right)-x\left(t-\tau_{2}\right)
\end{array}\right] \\
& \quad \leq 2 \xi_{t}^{T}\left[E_{1}, E_{5}, E_{6}+E_{7}\right] P\left[A_{c}, E_{1}-E_{3}, E_{3}-E_{4}\right]^{T} \xi_{t} \\
& =\xi_{t}^{T} \operatorname{SYM}\left(\left[E_{1}, E_{5}, E_{6}+E_{7}\right]\right. \\
& \left.\cdot P\left[A_{c}, E_{1}-E_{3}, E_{3}-E_{4}\right]^{T}\right) \xi_{t}, \\
& \dot{V}_{2}(t)=2 \sum_{i=1}^{n} \gamma_{i} f_{i}\left(x_{i}(t)\right) \dot{x}(t)=2 f^{T}(x(t)) \Gamma \dot{x}(t) \\
& \quad \leq 2 \xi_{t}^{T} E_{8} \Gamma A_{c}^{T} \xi_{t}, \\
& \dot{V}_{3}(t)=f^{T}(x(t)) S f(x(t))-(1-\dot{\tau}(t)) \\
& \cdot f^{T}(x(t-\tau(t))) S f(x(t-\tau(t))) \\
& +\left[x^{T}(t) x^{T}\left(t-\tau_{1}\right)\right] Q_{1}\left[x^{T}(t) x^{T}\left(t-\tau_{1}\right)\right]^{T} \\
& -(1-\dot{\tau}(t))\left[x^{T}(t) x^{T}(t-\tau(t))\right] Q_{1}\left[x^{T}(t)\right. \\
& \left.\cdot x^{T}(t-\tau(t))\right]^{T}
\end{aligned}
$$

$+2 \int_{t-\tau(t)}^{t-\tau_{1}} \frac{\partial\left[\begin{array}{ll}x^{T}(t) & x^{T}(s)\end{array}\right]}{\partial t} Q_{1}\left[\begin{array}{lll}x^{T}(t) & x^{T}(s)\end{array}\right]^{T} \mathrm{~d} s$

$\leq f^{T}(x(t)) S f(x(t))-(1-\mu) f^{T}(x(t-\tau(t)))$

$\cdot S f(x(t-\tau(t)))$

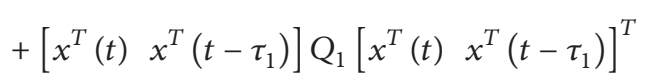

$-(1-\mu)\left[x^{T}(t) x^{T}(t-\tau(t))\right] Q_{1}\left[x^{T}(t)\right.$

$\left.\cdot x^{T}(t-\tau(t))\right]^{T}+2\left[\dot{x}^{T}(t), 0\right]$

$\cdot Q_{1} \int_{t-\tau(t)}^{t-\tau_{1}}\left[x^{T}(t) x^{T}(s)\right]^{T} \mathrm{~d} s \leq \xi_{t}^{T}\left\{E_{8} S E_{8}^{T}\right.$

$-(1-\mu) E_{9} S E_{9}^{T}+\left[E_{1}, E_{3}\right] Q_{1}\left[E_{1}, E_{3}\right]^{T}-(1-\mu)$

$\cdot\left[E_{1}, E_{2}\right] Q_{1}\left[E_{1}, E_{2}\right]^{T}+2\left[A_{c}, 0\right]$

- $\left.Q_{1}\left[\left(\tau(t)-\tau_{1}\right) E_{1}, E_{6}\right]^{T}\right\} \xi_{t}$,

$\dot{V}_{4}(t)=\left[\begin{array}{ll}x^{T}(t) & x^{T}(t)\end{array}\right] Q_{2}\left[\begin{array}{ll}x^{T}(t) & x^{T}(t)\end{array}\right]^{T}$

$+\left[\begin{array}{ll}x^{T}(t) & \left.x^{T}\left(t-\tau_{1}\right)\right]\end{array}\right] Q_{4}\left[\begin{array}{ll}x^{T}(t) & x^{T}\left(t-\tau_{1}\right)\end{array}\right]^{T}$

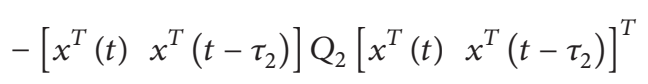

$-\left[\begin{array}{ll}x^{T}(t) & \left.x^{T}\left(t-\tau_{2}\right)\right]\end{array} Q_{4}\left[\begin{array}{ll}x^{T}(t) & x^{T}\left(t-\tau_{2}\right)\end{array}\right]^{T}\right.$

$+2\left[\dot{x}^{T}(t), 0\right] Q_{2} \int_{t-\tau_{2}}^{t}\left[\begin{array}{ll}x^{T}(t) & x^{T}(s)\end{array}\right]^{T} \mathrm{~d} s$

$+2\left[\dot{x}^{T}(t), 0\right] Q_{4} \int_{t-\tau_{2}}^{t-\tau_{1}}\left[\begin{array}{ll}x^{T}(t) & x^{T}(s)\end{array}\right]^{T} \mathrm{~d} s$

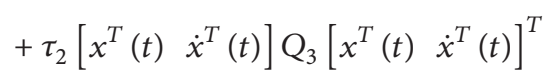

$+\tau_{21}\left[x^{T}\left(t-\tau_{1}\right) \dot{x}^{T}\left(t-\tau_{1}\right)\right] Q_{5}\left[x^{T}\left(t-\tau_{1}\right)\right.$

$\left.\cdot \dot{x}^{T}\left(t-\tau_{1}\right)\right]^{T}+\dot{x}^{T}(t)\left(\tau_{2}^{2} R_{1}+\tau_{2}^{3} R_{2}\right) \dot{x}(t)+\dot{x}^{T}(t$

$\left.-\tau_{1}\right)\left(\tau_{21}^{2} R_{3}+\tau_{21}^{3} R_{4}\right) \dot{x}\left(t-\tau_{1}\right)+V_{x}+V_{y}$

$=\xi_{t}^{T}\left\{\left[E_{1}, E_{1}\right] Q_{2}\left[E_{1}, E_{1}\right]^{T}+\left[E_{1}, E_{3}\right] Q_{4}\left[E_{1}, E_{3}\right]^{T}\right.$

$-\left[E_{1}, E_{4}\right]\left(Q_{2}+q_{4}\right)\left[E_{1}, E_{4}\right]^{T}+2\left[A_{c}, 0\right]$

$\cdot Q_{2}\left[\tau_{2} E_{1}, E_{5}+E_{6}+E_{7}\right]^{T}+2\left[A_{c}, 0\right]$

$\cdot Q_{4}\left[\left(\tau_{2}-\tau_{1}\right) E_{1}, E_{6}+E_{7}\right]^{T}+\tau_{2}\left[E_{1}, A_{c}\right]$

$\cdot Q_{3}\left[E_{1}, A_{c}\right]^{T}+\tau_{21}\left[E_{3}, E_{10}\right] Q_{5}\left[E_{3}, E_{10}\right]^{T}$

$\left.+A_{c}\left(\tau_{2}^{2} R_{1}+\tau_{2}^{3} R_{2}\right) A_{c}^{T}+E_{10}\left(\tau_{21}^{2} R_{3}+\tau_{21}^{3} R_{4}\right) E_{10}^{T}\right\}$

$\cdot \xi_{t}+V_{x}+V_{y}$, 
where

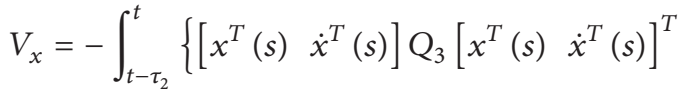

$$
\begin{aligned}
& +2\left(\tau_{2}-t+s\right) \dot{x}^{T}(s) R_{1} \dot{x}(s) \\
& \left.+3\left(\tau_{2}-t+s\right)^{2} \dot{x}^{T}(s) R_{2} \dot{x}(s)\right\} \mathrm{d} s,
\end{aligned}
$$

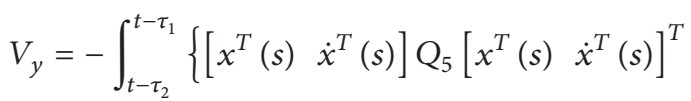

$$
\begin{aligned}
& +2\left(\tau_{2}-t+s\right) \dot{x}^{T}(s) R_{3} \dot{x}(s) \\
& \left.+3\left(\tau_{2}-t+s\right)^{2} \dot{x}^{T}(s) R_{4} \dot{x}(s)\right\} \mathrm{d} s .
\end{aligned}
$$

It is easy to obtain the following identities:

$$
\begin{aligned}
\tau_{2}-t+s= & (\tau(t)-t+s)+\left(\tau_{2}-\tau(t)\right), \\
\left(\tau_{2}-t+s\right)^{2}= & (\tau(t)-t+s)^{2}+\left(\tau_{2}^{2}-\tau^{2}(t)\right) \\
& +2\left(\tau_{2}-\tau(t)\right)(s-t) .
\end{aligned}
$$

Thus, we have

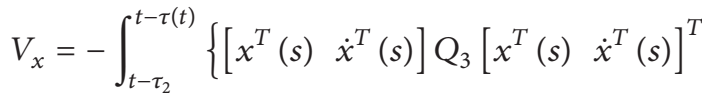

$$
\begin{aligned}
& +2\left(\tau_{2}-t+s\right) \dot{x}^{T}(s) R_{1} \dot{x}(s) \\
& \left.+3\left(\tau_{2}-t+s\right)^{2} \dot{x}^{T}(s) R_{2} \dot{x}(s)\right\} \mathrm{d} s
\end{aligned}
$$

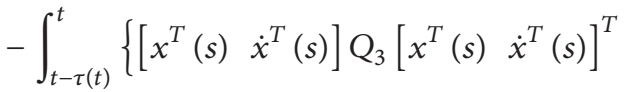

$$
\begin{aligned}
& +2\left(\tau_{2}-t+s\right) \dot{x}^{T}(s) R_{1} \dot{x}(s) \\
& \left.+3\left(\tau_{2}-t+s\right)^{2} \dot{x}^{T}(s) R_{2} \dot{x}(s)\right\} \mathrm{d} s
\end{aligned}
$$

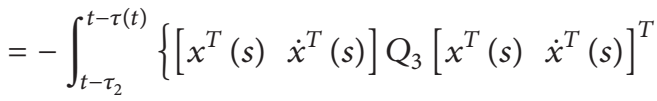

$$
\begin{aligned}
& +2\left(\tau_{2}-t+s\right) \dot{x}^{T}(s) R_{1} \dot{x}(s) \\
& \left.+3\left(\tau_{2}-t+s\right)^{2} \dot{x}^{T}(s) R_{2} \dot{x}(s)\right\} \mathrm{d} s \\
& -\int_{t-\tau(t)}^{t}\left\{\left[\begin{array}{ll}
x^{T}(s) & \dot{x}^{T}(s)
\end{array}\right] Q_{3}\left[\begin{array}{lll}
x^{T}(s) & \dot{x}^{T}(s)
\end{array}\right]^{T}\right. \\
& +2(\tau(t)-t+s) \dot{x}^{T}(s) R_{1} \dot{x}(s) \\
& \left.+3(\tau(t)-t+s)^{2} \dot{x}^{T}(s) R_{2} \dot{x}(s)\right\} \mathrm{d} s \\
& -\int_{t-\tau(t)}^{t} \dot{x}^{T}(s)\left[2\left(\tau_{2}-\tau(t)\right) R_{1}\right. \\
& \left.+3\left(\tau_{2}^{2}-\tau^{2}(t)\right) R_{2}+6\left(\tau_{2}-\tau(t)\right)(s-t) R_{2}\right] \\
& \cdot \dot{x}(s) \mathrm{d} s
\end{aligned}
$$

Similarly, we have

$$
\begin{aligned}
V_{y} & \leq-\int_{t-\tau_{2}}^{t-\tau(t)}\left\{\left[\begin{array}{lll}
x^{T}(s) & \dot{x}^{T}(s)
\end{array}\right] Q_{5}\left[\begin{array}{ll}
x^{T}(s) & \dot{x}^{T}(s)
\end{array}\right]^{T}\right. \\
& +2\left(\tau_{2}-t+s\right) \dot{x}^{T}(s) R_{3} \dot{x}(s) \\
& \left.+3\left(\tau_{2}-t+s\right)^{2} \dot{x}^{T}(s) R_{4} \dot{x}(s)\right\} \mathrm{d} s \\
& -\int_{t-\tau(t)}^{t-\tau_{1}}\left\{\left[x^{T}(s) \dot{x}^{T}(s)\right] Q_{5}\left[\begin{array}{ll}
x^{T}(s) & \dot{x}^{T}(s)
\end{array}\right]^{T}\right. \\
& +2(\tau(t)-t+s) \dot{x}^{T}(s) R_{3} \dot{x}(s) \\
& \left.+3(\tau(t)-t+s)^{2} \dot{x}^{T}(s) R_{4} \dot{x}(s)\right\} \mathrm{d} s .
\end{aligned}
$$

Applying Lemma 7 to $V_{x}$ and $V_{y}$, we get

$$
\begin{aligned}
V_{x} & \leq \xi_{t}^{T}\left\{\left(\tau_{2}-\tau(t)\right) F_{1}^{T} Q_{3}^{-1} F_{1}+2 F_{1}^{T}\left[E_{7}, E_{2}-E_{4}\right]^{T}\right. \\
& +\left(\tau_{2}-\tau(t)\right)^{2} F_{2}^{T} R_{1}^{-1} F_{2} \\
& +4 F_{2}^{T}\left[\left(\tau_{2}-\tau(t)\right) E_{2}-E_{7}\right]^{T}
\end{aligned}
$$




$$
\begin{aligned}
& +3\left(\tau_{2}-\tau(t)\right) F_{3}^{T} R_{2}^{-1} F_{3} \\
& +6 F_{3}^{T}\left[\left(\tau_{2}-\tau(t)\right) E_{2}-E_{7}\right]^{T}+\tau(t) F_{4}^{T} Q_{3}^{-1} F_{4} \\
& +2 F_{4}^{T}\left[E_{5}+E_{6}, E_{1}-E_{2}\right]^{T}+\tau^{2}(t) F_{5}^{T} R_{1}^{-1} F_{5} \\
& +4 F_{5}^{T}\left[\tau(t) E_{1}-\left(E_{5}+E_{6}\right)\right]^{T}+3 \tau(t) F_{6}^{T} R_{2}^{-1} F_{6} \\
& \left.+6 F_{6}^{T}\left[\tau(t) E_{1}-\left(E_{5}+E_{6}\right)\right]^{T}\right\} \xi_{t}, \\
V_{y} & \leq \xi_{t}^{T}\left\{\left(\tau_{2}-\tau(t)\right) F_{7}^{T} Q_{5}^{-1} F_{7}+2 F_{7}^{T}\left[E_{7}, E_{2}-E_{4}\right]^{T}\right. \\
& +\left(\tau_{2}-\tau(t)\right)^{2} F_{8}^{T} R_{3}^{-1} F_{8} \\
& +4 F_{8}^{T}\left[\left(\tau_{2}-\tau(t)\right) E_{2}-E_{7}\right]^{T} \\
& +3\left(\tau_{2}-\tau(t)\right) F_{9}^{T} R_{4}^{-1} F_{9} \\
& +6 F_{9}^{T}\left[\left(\tau_{2}-\tau(t)\right) E_{2}-E_{7}\right]^{T} \\
& +\left(\tau(t)-\tau_{1}\right) F_{10}^{T} Q_{5}^{-1} F_{10}+2 F_{10}^{T}\left[E_{6}, E_{3}-E_{2}\right]^{T} \\
& +\left(\tau(t)-\tau_{1}\right)^{2} F_{11}^{T} R_{3}^{-1} F_{11} \\
& +4 F_{11}^{T}\left[\left(\tau(t)-\tau_{1}\right) E_{3}-E_{6}\right]^{T} \\
& +3\left(\tau(t)-\tau_{1}\right) F_{12}^{T} R_{4}^{-1} F_{12} \\
& \left.+6 F_{12}^{T}\left[\left(\tau(t)-\tau_{1}\right) E_{3}-E_{6}\right]^{T}\right\} \xi_{t} .
\end{aligned}
$$

On the other hand, from assumption (H1), we have

$$
\begin{aligned}
- & 2\left(f_{i}\left(x_{i}(t)\right)-h_{i}^{-} x_{i}(t)\right) K_{1}\left(f_{i}\left(x_{i}(t)\right)-h_{i}^{+} x_{i}(t)\right) \\
\geq 0, & \\
- & 2\left(f_{i}\left(x_{i}(t-\tau(t))\right)-h_{i}^{-} x_{i}(t-\tau(t))\right) \\
\cdot & K_{1}\left(f_{i}\left(x_{i}(t-\tau(t))\right)-h_{i}^{+} x_{i}(t-\tau(t))\right) \geq 0,
\end{aligned}
$$

where $K_{1}=\operatorname{diag}\left(k_{11}, k_{12}, \ldots, k_{1 n}\right)>0, K_{2}=\operatorname{diag}\left(k_{21}, k_{22}\right.$, $\left.\ldots, k_{2 n}\right)>0, i=1,2, \ldots, n$.

From (27), it is easy to get that

$$
\begin{gathered}
2 \xi_{t}^{T}\left\{-E_{8} K_{1} E_{8}^{T}+E_{8} K_{1} H^{+} E_{1}^{T}+E_{1} H^{-} K_{1} E_{8}^{T}\right. \\
-E_{1} H^{-} K_{1} H^{+} E_{1}^{T}-E_{9} K_{2} E_{9}^{T}+E_{9} K_{2} H^{+} E_{2}^{T} \\
\left.+E_{2} H^{-} K_{2} E_{9}^{T}-E_{2} H^{-} K_{2} H^{+} E_{2}^{T}\right\} \geq 0 .
\end{gathered}
$$

It follows from (19)-(26) and (28) that

$$
\begin{aligned}
\dot{V}( & t) \leq \xi_{t}^{T}\left\{2\left[E_{1}, E_{5}, E_{6}+E_{7}\right]\right. \\
& \cdot P\left[A_{c}, E_{1}-E_{3}, E_{3}-E_{4}\right]^{T}+2 E_{8} \Gamma A_{c}^{T}+E_{8} S E_{8}^{T} \\
& -(1-\mu) E_{9} S E_{9}^{T}+\left[E_{1}, E_{3}\right] Q_{1}\left[E_{1}, E_{3}\right]^{T}-(1-\mu) \\
\cdot & {\left[E_{1}, E_{2}\right] Q_{1}\left[E_{1}, E_{2}\right]^{T}+2\left[A_{c}, 0\right] }
\end{aligned}
$$

$$
\begin{aligned}
& \text { - } Q_{1}\left[\left(\tau(t)-\tau_{1}\right) E_{1}, E_{6}\right]^{T}+\left[E_{1}, E_{1}\right] Q_{2}\left[E_{1}, E_{1}\right]^{T} \\
& +\left[E_{1}, E_{3}\right] Q_{4}\left[E_{1}, E_{3}\right]^{T}-\left[E_{1}, E_{4}\right]\left(Q_{2}+q_{4}\right) \\
& \cdot\left[E_{1}, E_{4}\right]^{T}+2\left[A_{c}, 0\right] Q_{2}\left[\tau_{2} E_{1}, E_{5}+E_{6}+E_{7}\right]^{T} \\
& +2\left[A_{c}, 0\right] Q_{4}\left[\tau_{21} E_{1}, E_{6}+E_{7}\right]^{T}+\tau_{2}\left[E_{1}, A_{c}\right] \\
& \cdot Q_{3}\left[E_{1}, A_{c}\right]^{T}+\tau_{21}\left[E_{3}, E_{10}\right] Q_{5}\left[E_{3}, E_{10}\right]^{T} \\
& +A_{c}\left(\tau_{2}^{2} R_{1}+\tau_{2}^{3} R_{2}\right) A_{c}^{T}+E_{10}\left(\tau_{21}^{2} R_{3}+\tau_{21}^{3} R_{4}\right) E_{10}^{T} \\
& +\left(\tau_{2}-\tau(t)\right) F_{1}^{T} Q_{3}^{-1} F_{1}+2 F_{1}^{T}\left[E_{7}, E_{2}-E_{4}\right]^{T} \\
& +\left(\tau_{2}-\tau(t)\right)^{2} F_{2}^{T} R_{1}^{-1} F_{2} \\
& +4 F_{2}^{T}\left[\left(\tau_{2}-\tau(t)\right) E_{2}-E_{7}\right]^{T}+3\left(\tau_{2}-\tau(t)\right) \\
& \text { - } F_{3}^{T} R_{2}^{-1} F_{3}+6 F_{3}^{T}\left[\left(\tau_{2}-\tau(t)\right) E_{2}-E_{7}\right]^{T}+\tau(t) \\
& \text { - } F_{4}^{T} Q_{3}^{-1} F_{4}+2 F_{4}^{T}\left[E_{5}+E_{6}, E_{1}-E_{2}\right]^{T}+\tau^{2}(t) \\
& \cdot F_{5}^{T} R_{1}^{-1} F_{5}+4 F_{5}^{T}\left[\tau(t) E_{1}-\left(E_{5}+E_{6}\right)\right]^{T}+3 \tau(t) \\
& \cdot F_{6}^{T} R_{2}^{-1} F_{6}+6 F_{6}^{T}\left[\tau(t) E_{1}-\left(E_{5}+E_{6}\right)\right]^{T} \\
& +\left(\tau_{2}-\tau(t)\right) F_{7}^{T} Q_{5}^{-1} F_{7}+2 F_{7}^{T}\left[E_{7}, E_{2}-E_{4}\right]^{T} \\
& +\left(\tau_{2}-\tau(t)\right)^{2} F_{8}^{T} R_{3}^{-1} F_{8} \\
& +4 F_{8}^{T}\left[\left(\tau_{2}-\tau(t)\right) E_{2}-E_{7}\right]^{T}+3\left(\tau_{2}-\tau(t)\right) \\
& \cdot F_{9}^{T} R_{4}^{-1} F_{9}+6 F_{9}^{T}\left[\left(\tau_{2}-\tau(t)\right) E_{2}-E_{7}\right]^{T} \\
& +\left(\tau(t)-\tau_{1}\right) F_{10}^{T} Q_{5}^{-1} F_{10}+2 F_{10}^{T}\left[E_{6}, E_{3}-E_{2}\right]^{T} \\
& +\left(\tau(t)-\tau_{1}\right)^{2} F_{11}^{T} R_{3}^{-1} F_{11} \\
& +4 F_{11}^{T}\left[\left(\tau(t)-\tau_{1}\right) E_{3}-E_{6}\right]^{T}+3\left(\tau(t)-\tau_{1}\right) \\
& \cdot F_{12}^{T} R_{4}^{-1} F_{12}+6 F_{12}^{T}\left[\left(\tau(t)-\tau_{1}\right) E_{3}-E_{6}\right]^{T} \\
& -2 E_{8} K_{1} E_{8}^{T}+2 E_{8} K_{1} H^{+} E_{1}^{T}+2 E_{1} H^{-} K_{1} E_{8}^{T} \\
& -2 E_{1} H^{-} K_{1} H^{+} E_{1}^{T}-2 E_{9} K_{2} E_{9}^{T}+2 E_{9} K_{2} H^{+} E_{2}^{T} \\
& \left.+2 E_{2} H^{-} K_{2} E_{9}^{T}-2 E_{2} H^{-} K_{2} H^{+} E_{2}^{T}\right\} \xi_{t}=\xi_{t}^{T}\left(\Delta_{0}\right. \\
& \left.+\Delta_{1}\right) \xi_{t},
\end{aligned}
$$

where $\Delta_{0}$ is defined in the theorem context, and

$$
\begin{aligned}
\Delta_{1}= & 2\left[A_{c}, 0\right] Q_{1}\left[\left(\tau(t)-\tau_{1}\right) E_{1}, E_{6}\right]^{T} \\
& +\left(\tau_{2}-\tau(t)\right) F_{1}^{T} Q_{3}^{-1} F_{1}+\left(\tau_{2}-\tau(t)\right)^{2} F_{2}^{T} R_{1}^{-1} F_{2} \\
& +4 F_{2}^{T}\left[\left(\tau_{2}-\tau(t)\right) E_{2}-E_{7}\right]^{T} \\
& +3\left(\tau_{2}-\tau(t)\right) F_{3}^{T} R_{2}^{-1} F_{3}
\end{aligned}
$$




$$
\begin{aligned}
& +6 F_{3}^{T}\left[\left(\tau_{2}-\tau(t)\right) E_{2}-E_{7}\right]^{T}+\tau(t) F_{4}^{T} Q_{3}^{-1} F_{4} \\
& +\tau^{2}(t) F_{5}^{T} R_{1}^{-1} F_{5}+4 F_{5}^{T}\left[\tau(t) E_{1}-\left(E_{5}+E_{6}\right)\right]^{T} \\
& +3 \tau(t) F_{6}^{T} R_{2}^{-1} F_{6}+6 F_{6}^{T}\left[\tau(t) E_{1}-\left(E_{5}+E_{6}\right)\right]^{T} \\
& +\left(\tau_{2}-\tau(t)\right) F_{7}^{T} Q_{5}^{-1} F_{7}+\left(\tau_{2}-\tau(t)\right)^{2} F_{8}^{T} R_{3}^{-1} F_{8} \\
& +4 F_{8}^{T}\left[\left(\tau_{2}-\tau(t)\right) E_{2}-E_{7}\right]^{T} \\
& +3\left(\tau_{2}-\tau(t)\right) F_{9}^{T} R_{4}^{-1} F_{9} \\
& +6 F_{9}^{T}\left[\left(\tau_{2}-\tau(t)\right) E_{2}-E_{7}\right]^{T} \\
& +\left(\tau(t)-\tau_{1}\right) F_{10}^{T} Q_{5}^{-1} F_{10} \\
& +\left(\tau(t)-\tau_{1}\right)^{2} F_{11}^{T} R_{3}^{-1} F_{11} \\
& +4 F_{11}^{T}\left[\left(\tau(t)-\tau_{1}\right) E_{3}-E_{6}\right]^{T} \\
& +3\left(\tau(t)-\tau_{1}\right) F_{12}^{T} R_{4}^{-1} F_{12} \\
& +6 F_{12}^{T}\left[\left(\tau(t)-\tau_{1}\right) E_{3}-E_{6}\right]^{T} .
\end{aligned}
$$

It is easy to see that $\Delta_{0}+\Delta_{1}$ is a quadratic convex combination of matrices on $\tau(t) \in\left[\tau_{1}, \tau_{2}\right]$.

Applying Lemma 5 to (16), we have

$$
\begin{aligned}
& \left.\left(\Delta_{0}+\Delta_{1}\right)\right|_{\tau(t)=\tau_{1}}=\Delta_{0}+\left.\Delta_{1}\right|_{\tau(t)=\tau_{1}}=\Delta_{0}+2\left(\tau_{21} E_{2}\right. \\
& \left.\quad-E_{7}\right)\left(2 F_{2}+3 F_{3}+2 F_{8}+3 F_{9}\right)+2\left(\tau_{1} E_{1}\right. \\
& \left.\quad-\left(E_{1}+E_{5}\right)\right)\left(2 F_{5}+3 F_{6}\right)+\tau_{1}\left(F_{4}^{T} Q_{3}^{-1} F_{4}\right. \\
& \left.\quad+3 F_{6}^{T} R_{2}^{-1} F_{6}\right)+\tau_{1}^{2} F_{5}^{T} R_{1}^{-1} F_{5}+\tau_{21}\left(F_{1}^{T} Q_{3}^{-1} F_{1}\right. \\
& \left.+F_{7}^{T} Q_{5}^{-1} F_{7}+3 F_{3}^{T} R_{2}^{-1} F_{3}+3 F_{9}^{T} R_{4}^{-1} F_{9}\right) \\
& +\tau_{21}^{2}\left(F_{2}^{T} R_{1}^{-1} F_{2}+F_{8}^{T} R_{3}^{-1} F_{8}\right)<0, \\
& \left.\left(\Delta_{0}+\Delta_{1}\right)\right|_{\tau(t)=\tau_{2}}=\Delta_{0}+\left.\Delta_{1}\right|_{\tau(t)=\tau_{2}}=\Delta_{0}+2\left[A_{c}, 0\right] \\
& +Q_{1}\left[\tau_{21} E_{1}, E_{6}\right]^{T}-2 E_{7}\left(2 F_{2}+3 F_{3}+2 F_{8}+3 F_{9}\right) \\
& +2\left(\tau_{2} E_{1}-\left(E_{5}+E_{6}\right)\right)\left(2 F_{5}+3 F_{6}\right)+\tau_{2}\left(F_{4}^{T} Q_{3}^{-1} F_{4}\right. \\
& \left.+3 F_{6}^{T} R_{2}^{-1} F_{6}\right)+\tau_{2}^{2} F_{5}^{T} R_{1}^{-1} F_{5}<0 .
\end{aligned}
$$

Since $\xi_{t}^{T}\left\{F_{2}^{T} R_{1}^{-1} F_{2}+F_{5}^{T} R_{1}^{-1} F_{5}+F_{8}^{T} R_{3}^{-1} F_{8}+F_{11}^{T} R_{3}^{-1} F_{11}\right\} \xi_{t} \geq$ 0 , from Lemma 6, if LMIs (16) are true, then $\Delta_{0}+\Delta_{1}<$ $0, \forall \tau(t) \in\left[\tau_{1}, \tau_{2}\right]$. Then, we can see that the origin of system (1) is asymptotically stable.

The proof is completed.

Remark 9. In [16], stability analysis for neural networks with time-varying delay is studied by using quadratic convex combination. Our results have two advantages compared with the results of that paper. On the one hand, the information on the lower bound $\tau_{1}$ of the time-varying delays is considered.
On the other hand, the augmented vector $\zeta(t)$ includes the distributed delay terms.

Remark 10. We use three inequalities in Lemma 7 combined with the quadratic convex combination implied by Lemma 6, rather than Jensen's inequality and the linear convex combination. In addition, our theoretical proof is not concerned with free-weighting matrix method.

Remark 11. To use the quadratic convex approach, we construct the Lyapunov-Krasovskii functional with the following term: $\int_{t-\tau_{2}}^{t} \sum_{j=1}^{3}\left(\tau_{2}+t-s\right)^{j} g(s) \mathrm{d} s$. The degree increase of $\tau_{2}-t+s$ by 1 means the number increase of the integral by 1 due to the fact that $\int_{\tau_{2}}^{t} \int_{t+\theta}^{t} g(s) \mathrm{d} s \mathrm{~d} \theta=\int_{t-\tau_{2}}^{t}\left(\tau_{2}-t+s\right) g(s) \mathrm{d} s$.

In the case $\tau_{1}=0$, we have the following result.

Corollary 12. Suppose assumptions (H1) and (H2) hold with $\tau_{1}=0$; the origin of system (1) is globally asymptotically stable if there exist matrices $P>0, S>0, Q_{i}>0(i=1,2,3)$, $R_{j}>0(j=1,2)$, and $\Gamma=\operatorname{diag}\left(\gamma_{1}, \gamma_{2}, \ldots, \gamma_{n}\right)>0, K_{1}=$ $\operatorname{diag}\left(k_{11}, k_{12}, \ldots, k_{1 n}\right)>0, K_{2}=\operatorname{diag}\left(k_{21}, k_{22}, \ldots, k_{2 n}\right)>0$, and $F_{i}(i=1,2, \ldots, 6)$, such that the following two LMIs hold:

$$
\begin{aligned}
& \Xi=\left[\begin{array}{cccc}
\Delta_{0} & \tau_{2} F_{1}^{T} & \sqrt{3} \tau_{2} F_{3}^{T} & \tau_{2} F_{2}^{T} \\
* & -\tau_{2} Q_{3} & 0 & 0 \\
* & * & -\tau_{2} R_{2} & 0 \\
* & * & * & -R_{1}
\end{array}\right]<0, \\
& \Pi=\left[\begin{array}{cccc}
\Pi_{11} & \tau_{2} F_{4}^{T} & \sqrt{3} \tau_{2} F_{6}^{T} & \tau_{2} F_{5}^{T} \\
* & -\tau_{2} Q_{3} & 0 & 0 \\
* & * & -\tau_{2} R_{2} & 0 \\
* & * & * & -R_{1}
\end{array}\right]<0,
\end{aligned}
$$

where $\Pi_{11}=\Delta_{0}+\tau_{2} \operatorname{SYM}\left(\left[A_{c}, 0\right] Q_{1}\left[E_{1}, 0\right]^{T}\right)-$ $\tau_{2} \operatorname{SYM}\left(E_{2}\left(2 F_{2}+3 F_{3}\right)\right)+\tau_{2} \operatorname{SYM}\left(E_{1}\left(2 F_{5}+3 F_{6}\right)\right)$ with

$$
\begin{aligned}
\Delta_{0} & =\operatorname{SYM}\left(\left[E_{1}, E_{4}+E_{5}\right] P\left[A_{c}, E_{1}-E_{3}\right]^{T}+E_{6} \Gamma A_{c}^{T}\right) \\
& +E_{6} S E_{6}^{T}-(1-\mu) E_{7} S E_{7}^{T}+\left[E_{1}, E_{1}\right]\left(Q_{1}+Q_{2}\right) \\
& \cdot\left[E_{1}, E_{1}\right]^{T}-(1-\mu)\left[E_{1}, E_{2}\right] Q_{1}\left[E_{1}, E_{2}\right]^{T} \\
& -\left[E_{1}, E_{3}\right] Q_{2}\left[E_{1}, E_{3}\right]^{T}+\operatorname{SYM}\left(\left[A_{c}, 0\right] Q_{1}\left[0, E_{5}\right]^{T}\right. \\
& \left.+\left[A_{c}, 0\right] Q_{2}\left[\tau_{2} E_{1}, E_{4}+E_{5}\right]^{T}\right)+\tau_{2}\left[E_{1}, A_{c}\right] \\
& \cdot Q_{3}\left[E_{1}, A_{c}\right]^{T}+A_{c}\left(\tau_{2}^{2} R_{1}+\tau_{2}^{3} R_{2}\right) A_{c}^{T} \\
& +\operatorname{SYM}\left(\left[E_{5}, E_{1}-E_{2}\right] F_{1}\right. \\
& \left.+\left(\tau_{2} E_{2}-E_{5}\right)\left(2 F_{2}+3 F_{3}\right)+\left[E_{4}, E_{1}-E_{2}\right] F_{4}\right) \\
& -\operatorname{SYM}\left(E_{4}\left(2 F_{5}+3 F_{6}\right)\right)+\operatorname{SYM}\left(E_{8} K_{1} H^{+} E_{1}^{T}\right. \\
& \left.+E_{1} H^{-} K_{1} E_{8}^{T}+E_{9} K_{2} H^{+} E_{2}^{T}+E_{2} H^{-} K_{2} E_{9}^{T}\right)
\end{aligned}
$$




$$
\begin{aligned}
& -\operatorname{SYM}\left(E_{8} K_{1} E_{8}^{T}+E_{1} H^{-} K_{1} H^{+} E_{1}^{T}+E_{9} K_{2} E_{9}^{T}\right. \\
& \left.+E_{2} H^{-} K_{2} H^{+} E_{2}^{T}\right) .
\end{aligned}
$$

In addition, when the information of the time derivative of delays is unknown or the derivative of the delays does not exist, then we have the following result.

Corollary 13. Suppose assumption (H1) holds with $\tau_{1}=0$; the origin of system (1) is globally asymptotically stable if there exist matrices $P>0, Q_{i}>0(i=2,3), R_{j}>0(j=1,2)$, and $\Gamma=\operatorname{diag}\left(\gamma_{1}, \gamma_{2}, \ldots, \gamma_{n}\right)>0, K_{1}=\operatorname{diag}\left(k_{11}, k_{12}, \ldots, k_{1 n}\right)>0$, $K_{2}=\operatorname{diag}\left(k_{21}, k_{22}, \ldots, k_{2 n}\right)>0$, and $F_{i}(i=1,2, \ldots, 6)$, such that the following two LMIs hold:

$$
\begin{aligned}
& \Xi=\left[\begin{array}{cccc}
\Delta_{0} & \tau_{2} F_{1}^{T} & \sqrt{3} \tau_{2} F_{3}^{T} & \tau_{2} F_{2}^{T} \\
* & -\tau_{2} Q_{3} & 0 & 0 \\
* & * & -\tau_{2} R_{2} & 0 \\
* & * & * & -R_{1}
\end{array}\right]<0, \\
& \Pi=\left[\begin{array}{cccc}
\Pi_{11} & \tau_{2} F_{4}^{T} & \sqrt{3} \tau_{2} F_{6}^{T} & \tau_{2} F_{5}^{T} \\
* & -\tau_{2} Q_{3} & 0 & 0 \\
* & * & -\tau_{2} R_{2} & 0 \\
* & * & * & -R_{1}
\end{array}\right]<0,
\end{aligned}
$$

where $\Pi_{11}=\Delta_{0}+\tau_{2} \operatorname{SYM}\left(\left[A_{c}, 0\right] Q_{1}\left[E_{1}, 0\right]^{T}\right)-$ $\tau_{2} \operatorname{SYM}\left(E_{2}\left(2 F_{2}+3 F_{3}\right)\right)+\tau_{2} \operatorname{SYM}\left(E_{1}\left(2 F_{5}+3 F_{6}\right)\right)$ with

$$
\begin{aligned}
\Delta_{0} & =\operatorname{SYM}\left(\left[E_{1}, E_{4}+E_{5}\right] P\left[A_{c}, E_{1}-E_{3}\right]^{T}+E_{6} \Gamma A_{c}^{T}\right) \\
& +\left[E_{1}, E_{1}\right] Q_{2}\left[E_{1}, E_{1}\right]^{T}-\left[E_{1}, E_{3}\right] Q_{2}\left[E_{1}, E_{3}\right]^{T} \\
& +\operatorname{SYM}\left(\left[A_{c}, 0\right] Q_{1}\left[0, E_{5}\right]^{T}\right. \\
& \left.+\left[A_{c}, 0\right] Q_{2}\left[\tau_{2} E_{1}, E_{4}+E_{5}\right]^{T}\right)+\tau_{2}\left[E_{1}, A_{c}\right] \\
& +Q_{3}\left[E_{1}, A_{c}\right]^{T}+A_{c}\left(\tau_{2}^{2} R_{1}+\tau_{2}^{3} R_{2}\right) A_{c}^{T} \\
& +\operatorname{SYM}\left(\left[E_{5}, E_{1}-E_{2}\right] F_{1}\right. \\
& \left.+\left(\tau_{2} E_{2}-E_{5}\right)\left(2 F_{2}+3 F_{3}\right)+\left[E_{4}, E_{1}-E_{2}\right] F_{4}\right) \\
& -\operatorname{SYM}\left(E_{4}\left(2 F_{5}+3 F_{6}\right)\right)+\operatorname{SYM}\left(E_{8} K_{1} H^{+} E_{1}^{T}\right. \\
& \left.+E_{1} H^{-} K_{1} E_{8}^{T}+E_{9} K_{2} H^{+} E_{2}^{T}+E_{2} H^{-} K_{2} E_{9}^{T}\right) \\
& -\operatorname{SYM}\left(E_{8} K_{1} E_{8}^{T}+E_{1} H^{-} K_{1} H^{+} E_{1}^{T}+E_{9} K_{2} E_{9}^{T}\right. \\
& \left.+E_{2} H^{-} K_{2} H^{+} E_{2}^{T}\right) .
\end{aligned}
$$

Remark 14. It is worth noting that when we consider system (1) without switching, that is, $d_{i}^{*}=d_{i}^{* *}, a_{i j}^{*}=a_{i j}^{* *}$, and $b_{i j}^{*}=$ $b_{i j}^{* *}$, then Corollary 12 is the main Theorem 1 of [16].

Remark 15. Compared with the results on stability of neural networks with continuous right-hand side [6], our results on stability of neural networks are with discontinuous righthand sides. So the results of this paper are less conservative and more general.

\section{Numerical Example}

In this section, an example is provided to verify the effectiveness of the results obtained in the previous section.

Example 1. Consider two-dimensional switched neural networks with time-varying delays

$$
\begin{aligned}
\dot{x}_{i}(t)= & -d_{i}\left(x_{i}(t)\right) x_{i}(t)+\sum_{j=1}^{2} a_{i j}\left(x_{i}(t)\right) f_{j}\left(x_{j}(t)\right) \\
& +\sum_{j=1}^{2} b_{i j}\left(x_{i}(t)\right) f_{j}\left(x_{j}\left(t-\tau_{j}(t)\right)\right),
\end{aligned}
$$

$$
t \geq 0, i=1,2,
$$

where

$$
\begin{aligned}
& d_{1}\left(x_{1}(t)\right)= \begin{cases}2, & x_{1}(t) \leq 0, \\
2.1, & x_{1}(t)>0,\end{cases} \\
& d_{2}\left(x_{2}(t)\right)= \begin{cases}2.1, & x_{2}(t) \leq 0, \\
2, & x_{2}(t)>0,\end{cases} \\
& a_{11}\left(x_{1}(t)\right)= \begin{cases}1, & x_{1}(t) \leq 0, \\
0.9, & x_{1}(t)>0,\end{cases} \\
& a_{12}\left(x_{1}(t)\right)= \begin{cases}1, & x_{1}(t) \leq 0, \\
1.2, & x_{1}(t)>0,\end{cases} \\
& a_{21}\left(x_{2}(t)\right)= \begin{cases}-0.9, & x_{2}(t) \leq 0, \\
-1, & x_{2}(t)>0,\end{cases} \\
& a_{22}\left(x_{2}(t)\right)= \begin{cases}-1.2, & x_{2}(t) \leq 0, \\
-1, & x_{2}(t)>0,\end{cases} \\
& b_{11}\left(x_{1}(t)\right)= \begin{cases}-0.7, & x_{1}(t) \leq 0, \\
-0.8, & x_{1}(t)>0,\end{cases} \\
& b_{12}\left(x_{1}(t)\right)= \begin{cases}1, & x_{1}(t) \leq 0, \\
1.2, & x_{1}(t)>0,\end{cases} \\
& b_{21}\left(x_{2}(t)\right)= \begin{cases}1, & x_{2}(t) \leq 0, \\
0.9, & x_{2}(t)>0,\end{cases} \\
& b_{22}\left(x_{2}(t)\right)= \begin{cases}-1, & x_{2}(t) \leq 0, \\
-1.2, & x_{2}(t)>0,\end{cases}
\end{aligned}
$$


with $\tau_{j}(t)=0.5 \cos (1.6 t)+3, j=1,2$, and take the activation function as $f(x)=\left(\sin \left(x_{1}\right), \sin \left(x_{2}\right)\right)^{T}$. We can obtain that $\tau_{1}=2.5, \tau_{2}=3.5, \tau_{21}=1, \mu=0.8$,

$$
\begin{aligned}
D & =\left[\begin{array}{ll}
2 & 0 \\
0 & 2
\end{array}\right], \\
A & =\left[\begin{array}{ll}
1 & 1.2 \\
1 & 1.2
\end{array}\right], \\
B & =\left[\begin{array}{ll}
0.8 & 1.2 \\
1 & 1.2
\end{array}\right] \\
H^{-} & =\left[\begin{array}{ll}
-1 & 0 \\
0 & -1
\end{array}\right], \\
H^{+} & =\left[\begin{array}{ll}
1 & 0 \\
0 & 1
\end{array}\right] .
\end{aligned}
$$

For written simplification, we use the Matlab LMI Control Toolbox and then a solution to LMIs (32) is obtained $\left(F_{i}, i=\right.$ $1, \ldots, 6$, is omitted since the dimension is too big) as follows:

$$
\begin{aligned}
& P=\left[\begin{array}{cccc}
28.5171 & -20.9241 & 0.5484 & -0.4280 \\
-20.9241 & 27.7701 & -0.4064 & 0.4984 \\
0.5484 & -0.4064 & 10.8451 & -3.9493 \\
-0.4280 & 0.4984 & -3.9493 & 10.6288
\end{array}\right], \\
& Q_{1}=\left[\begin{array}{cccc}
7.3594 & -1.0832 & 0.0588 & -0.0060 \\
-1.0832 & 7.4606 & -0.0074 & 0.0723 \\
0.0588 & -0.0074 & 3.5541 & -0.4045 \\
-0.0060 & 0.0723 & -0.4045 & 3.6751
\end{array}\right] \text {, } \\
& Q_{2}=\left[\begin{array}{cccc}
67.4363 & -64.0296 & 3.2893 & -3.0414 \\
-64.0296 & 65.2276 & -3.0862 & 0.0723 \\
3.2893 & -3.0862 & 35.1371 & -5.2829 \\
-3.0414 & 0.0723 & -5.2829 & 34.8395
\end{array}\right] \text {, } \\
& Q_{3}=\left[\begin{array}{cccc}
15.7736 & -2.2877 & 0.8863 & -0.8539 \\
-2.2877 & 15.6801 & -0.8085 & 0.4984 \\
0.8863 & -0.8085 & 7.6796 & -6.7268 \\
-0.8539 & 0.4984 & -6.7268 & 7.3682
\end{array}\right], \\
& S=\left[\begin{array}{ll}
2.4075 & 1.0722 \\
1.0722 & 2.5766
\end{array}\right] \text {, } \\
& G=\left[\begin{array}{cc}
3.5681 & 0 \\
0 & 3.7071
\end{array}\right] \text {, }
\end{aligned}
$$

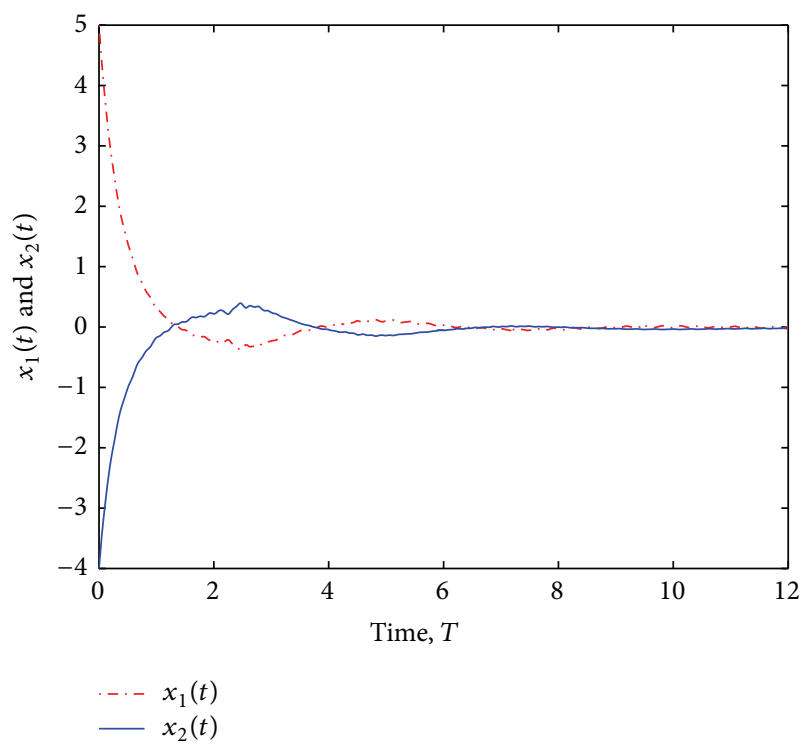

FIgURE 1: The state curves of system (36).

$$
\begin{aligned}
& K_{1}=\left[\begin{array}{cc}
57.4100 & 0 \\
0 & 65.2979
\end{array}\right], \\
& K_{2}=\left[\begin{array}{cc}
26.5401 & 0 \\
0 & 29.9701
\end{array}\right] .
\end{aligned}
$$

Therefore, according to Corollary 12, we can see that the origin of system (36) is globally asymptotically stable. The state trajectories of variables $x_{1}(t)$ and $x_{2}(t)$ are shown in Figure 1.

Remark 16. Because the parameters of system (1) are discontinuous, the results obtained in [6] about neural networks with continuous right-hand sides cannot be used here. In addition, the lower bounds of the delays of system (36) are not zero, so the results obtained in $[8,9,16]$ cannot be used here.

\section{Conclusions}

In this paper, the delay-dependent stability for a class of switched neural networks with time-varying delays has been studied by using the quadratic convex combination. Some delay-dependent criteria in terms of LMIs have been obtained. The lower bound $\tau_{1}$ of the time-varying delays is considered to be nonzero so that the information of $\tau_{1}$ can be used adequately. It is worth noting that we resort to neither Jensen's inequality with delay-dividing approach nor the freeweighting matrix method compared with previous results.

\section{Conflict of Interests}

The authors declare that there is no conflict of interests regarding the publication of this paper. 


\section{Acknowledgments}

The authors would like to thank the financial supports from the National Natural Science Foundation of China (nos. 61304068 and 61473334), Jiangsu Qing Lan Project, and PAPD.

\section{References}

[1] A. Cichocki and R. Unbehauen, Neural Networks for Optimization and Signal Processing, Wiley, New York, NY, USA, 1993.

[2] L. O. Chua and L. Yang, "Cellular neural networks: applications," IEEE Transactions on Circuits and Systems, vol. 35, no. 10, pp. 1273-1290, 1988.

[3] Z. Zeng and J. Wang, "Analysis and design of associative memories based on recurrent neural networks with linear saturation activation functions and time-varying delays," Neural Computation, vol. 19, no. 8, pp. 2149-2182, 2007.

[4] X. Liao and S. Guo, "Delay-dependent asymptotic stability of Cohen-Grossberg models with multiple time-varying delays," Discrete Dynamics in Nature and Society, vol. 2007, Article ID 28960, 17 pages, 2007.

[5] Q. Zhu, J. Cao, and R. Rakkiyappan, "Exponential input-to-state stability of stochastic Cohen-Grossberg neural networks with mixed delays," Nonlinear Dynamics, vol. 79, no. 2, pp. 1085-1098, 2015.

[6] Q. Zhu, R. Rakkiyappan, and A. Chandrasekar, "Stochastic stability of Markovian jump BAM neural networks with leakage delays and impulse control," Neurocomputing, vol. 136, pp. 136151, 2014.

[7] Y. Shen and J. Wang, "Almost sure exponential stability of recurrent neural networks with Markovian switching," IEEE Transactions on Neural Networks, vol. 20, no. 5, pp. 840-855, 2009.

[8] O.-M. Kwon, M.-J. Park, S.-M. Lee, J. H. Park, and E.-J. Cha, "Stability for neural networks with time-varying delays via some new approaches," IEEE Transactions on Neural Networks and Learning Systems, vol. 24, no. 2, pp. 181-193, 2013.

[9] H. Zhang, Z. Liu, G.-B. Huang, and Z. Wang, "Novel weighting delay-based stability criteria for recurrent neural networks with time varying delay," IEEE Transactions on Neural Networks, vol. 21, no. 1, pp. 91-106, 2010.

[10] J. Sun, G. P. Liu, J. Chen, and D. Rees, "Improved delay-rangedependent stability criteria for linear systems with time-varying delays," Automatica, vol. 46, no. 2, pp. 466-470, 2010.

[11] E. Fridman, U. Shaked, and K. Liu, "New conditions for delayderivative-dependent stability," Automatica, vol. 45, no. 11, pp. 2723-2727, 2009.

[12] H. Yu, X. Yang, C. Wu, and Q. Zeng, "Stability analysis for delayed neural networks: reciprocally convex approach," Mathematical Problems in Engineering, vol. 2013, Article ID 639219, 12 pages, 2013.

[13] P. G. Park, J. W. Ko, and C. Jeong, "Reciprocally convex approach to stability of systems with time-varying delays," Automatica, vol. 47, no. 1, pp. 235-238, 2011.

[14] T. Li, X. Yang, P. Yang, and S. Fei, "New delay-variationdependent stability for neural networks with time-varying delay," Neurocomputing, vol. 101, pp. 361-369, 2013.

[15] J.-H. Kim, "Note on stability of linear systems with time-varying delay," Automatica, vol. 47, no. 9, pp. 2118-2121, 2011.
[16] H. G. Zhang, F. S. Yang, X. D. Liu, and Q. L. Zhang, "Stability analysis for neural networks with time-varying delay based on quadratic convex combination," IEEE Transactions on Neural Networks and Learning Systems, vol. 24, no. 4, pp. 513-521, 2013.

[17] X.-M. Zhang and Q.-L. Han, "Global asymptotic stability analysis for delayed neural networks using a matrix-based quadratic convex approach," Neural Networks, vol. 54, pp. 57-69, 2014.

[18] A. F. Filippov, Differential Equations with Discontinuous Righthand Sides, vol. 18 of Mathematics and its Applications, Kluwer Academic, Dordrecht, The Netherlands, 1988.

[19] F. H. Clarke, Y. S. Ledyaev, R. J. Stern, and P. R. Wolenski, Nonsmooth Analysis and Control Theory, vol. 178 of Graduate Texts in Mathematics, Springer, New York, NY, USA, 1998.

[20] J.-P. Aubin and A. Cellina, Differential Inclusions, Springer, Berlin, Germany, 1984.

[21] S. Boyd, L. El Ghaoui, E. Feron, and V. Balakrishnan, Linear Matrix Inequalities in System and Control Theory, SIAM, Philadelphia, Pa, USA, 1994. 


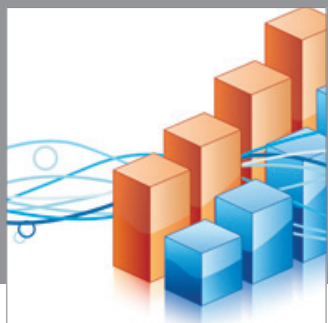

Advances in

Operations Research

mansans

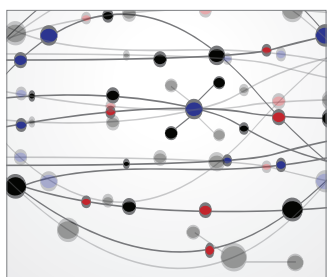

The Scientific World Journal
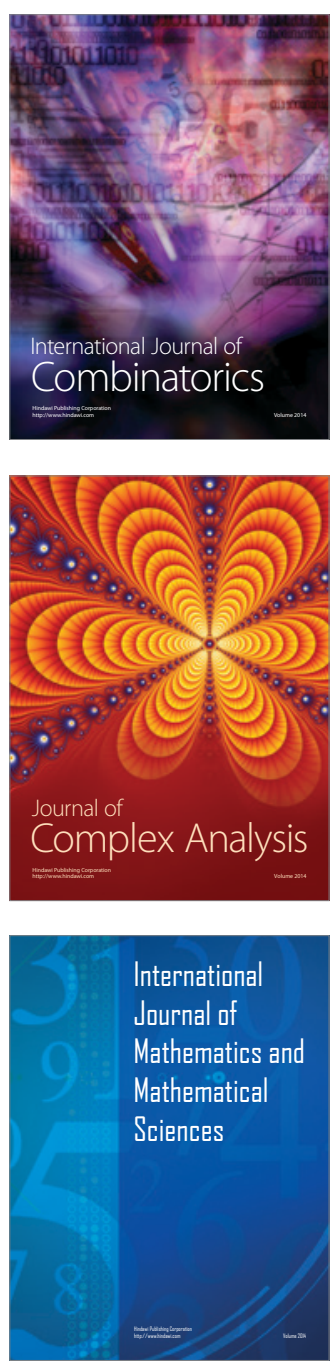
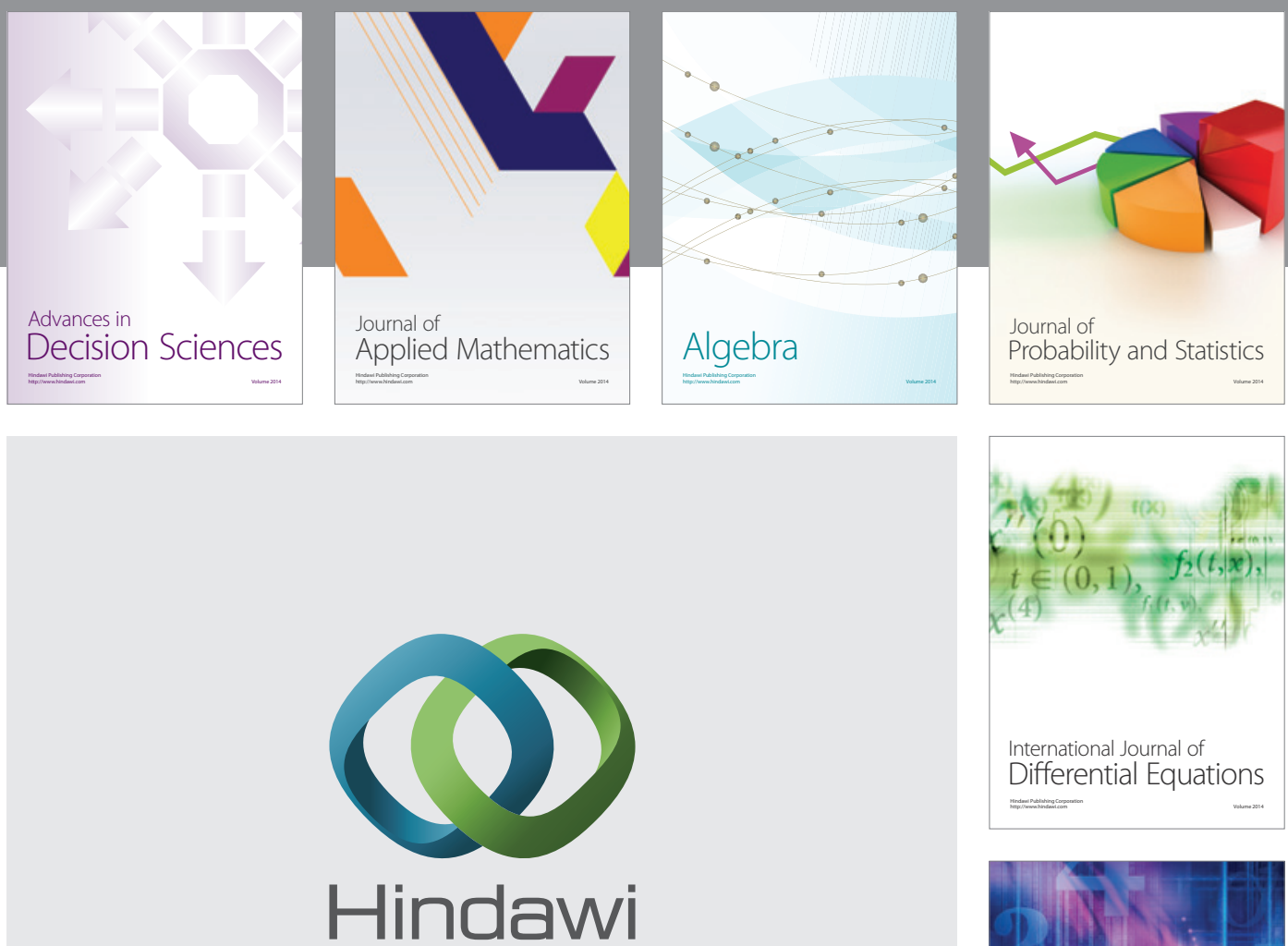

Submit your manuscripts at http://www.hindawi.com
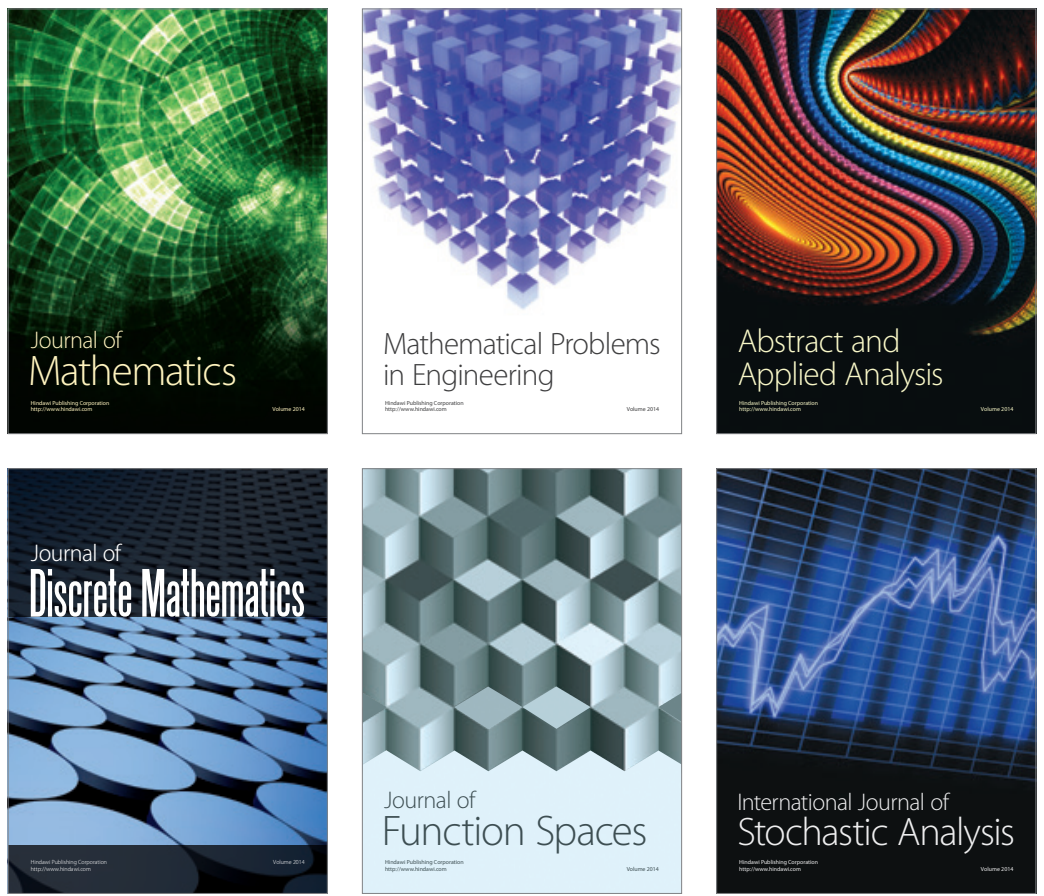

Journal of

Function Spaces

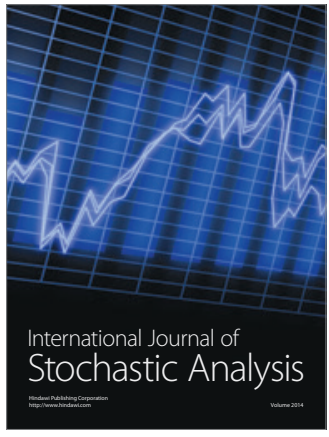

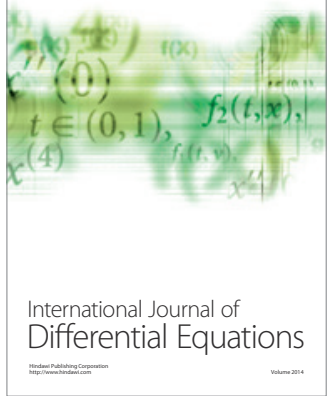
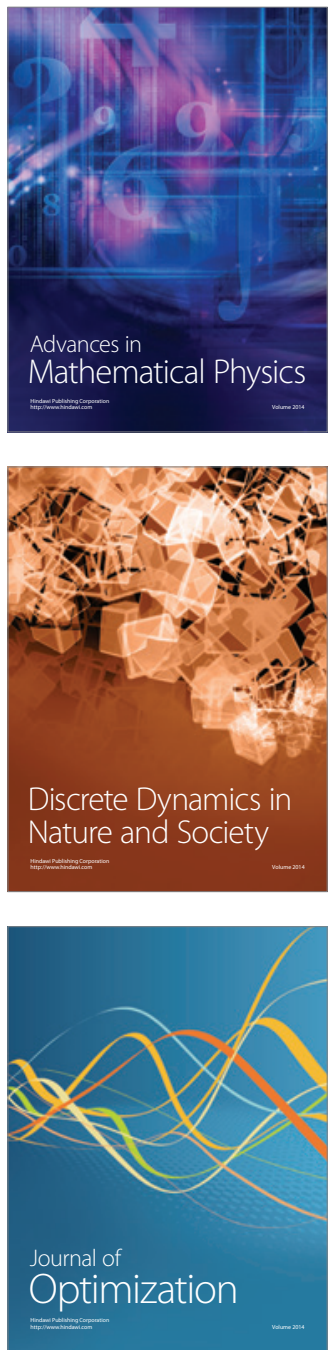